\title{
The Semantic Web: Suppliers and Customers
}

\author{
Rudi Studer \\ Institute AIFB, Universität Karlsruhe (TH) \\ D-76128 Karlsruhe, Germany \\ studer@aifb.uni-karlsruhe.de
}

\begin{abstract}
The notion of the Semantic Web can be coined as a Web of data when bringing database content to the Web or as a Web of enriched human-readable content when encoding the semantics of web-resources in a machine-interpretable form.

It has been clear from the beginning that realizing the Semantic Web vision will require interdisciplinary research. At this the fifth ISWC, it is time to re-examine the extent to which interdisciplinary work has played and can play a role in Semantic Web research, and even how Semantic Web research can contribute to other disciplines. Core Semantic Web research has drawn from various disciplines, such as knowledge representation and formal ontologies, reusing and further developing their techniques in a new context.

However, there are several other disciplines that explore research issues very relevant to the Semantic Web in different guises and to differing extents. As a community, we can benefit by also recognizing and drawing from the research in these different disciplines. On the other hand, Semantic Web research also has much to contribute to these disciplines and communities. For example, the Semantic Web offers scenario that often ask for unprecedented scalability of techniques from other disciplines. Throughout the talk, I will illustrate these points through examples from disciplines such as natural language processing, databases, software engineering and automated reasoning.

The industry also has a major role to play in the realization of the Semantic Web vision. I will therefore additionally examine the added value of Semantic Web technologies for commercial applications and discuss issues that should be addressed for broadening the market for Semantic Web technologies.
\end{abstract}

\section{About the Speaker}

Rudi Studer is Full Professor in Applied Informatics at the University of Karlsruhe, Institute AIFB (www.aifb.uni-karlsruhe.de/WBS). His research interests include knowledge management, Semantic Web technologies and applications, ontology management, data and text mining, service-oriented architectures, peer-to-peer systems, and Semantic Grid.

Rudi Studer is also director in the research department Information Process Engineering at the FZI Research Center for Information Technologies at the 
University of Karlsruhe (www.fzi.de/ipe) and one of the presidents of the FZI Research Center, as well as co-founder of the spin-off company ontoprise $\mathrm{GmbH}$ (www.ontoprise.de) that develops semantic applications. He is the current president of the Semantic Web Science Association (www.iswsa.org) and Editor-inchief of the journal Web Semantics: Science, Services, and Agents on the World Wide Web (www.websemanticsjournal.org ).

He is also engaged in various national and international cooperation projects being funded by various agencies such as Deutsche Forschungsgemeinschaft (DFG), the European Commission, the German Ministry of Education and Research, and by industry. 